\title{
A Lightweight Currency-based P2P VoD Incentive Mechanism
}

\author{
Chi Wang, Hongbo Wang, Yu Lin \\ State Key laboratory of Networking and Switching \\ Technology, \\ Beijing University of Posts and Telecommunications, \\ Beijing 100876, China \\ chiziwang@gmail.com, \{hbwang, linyu\}@bupt.edu.cn
}

\author{
Shanzhi Chen \\ State key Laboratory of Wireless Mobile \\ Communication, \\ China Academy of Telecommunication Technology, \\ Beijing 100083, China \\ chenshanzhi@yahoo.com.cn
}

\begin{abstract}
Users in a Peer-to-Peer Video on Demand (P2P VoD) system are allowed to watch any video file at any point in time and to use VCR operations like fast forward, rewind and pause at any time, which decreases their ability to help each other and offload the server. Encouraging peers to stay in the system longer and share the video files they have downloaded completely can greatly increase peers' cooperation and alleviate server's load. So we propose a lightweight incentive mechanism based on virtual currency to improve the performance of the system. If a peer shares a video file which he has completely downloaded before, he will get paid by the audience peers who are in the process of watching that video. By neglecting the data exchanges among audience peers in the same video channel, we decrease the cost aroused by the incentive mechanism, and stimulate peers to share the video files they have already downloaded completely which are more stable than the video files in the watching process. Game theoretic analysis proves that the proposed incentive mechanism can significantly relieve server's heavy load and increase users' contributions.
\end{abstract}

Keywords- peer-to-peer, Video on Demand, incentive, virtual currency, lightweight

\section{INTRODUCTION}

Peer-to-peer (P2P) is very popular in the Internet because of its powerful sharing and distributing ability. Users share files or resources with each other in a P2P system, but they have strong motivations to be free riding if the system lacks of suitable incentive mechanism that stimulates sharing. Such behavior is observed in existing peer-to-peer systems, for example, the study of Gnutella shows that 85 percent of users share no files [1].

Early P2P incentive is based on barter-exchange, which means that a peer offers data needed for another peer and gets data he needs as a reward. Tit-for-tat which is widely used in BT [2] is a typical barter-exchange incentive mechanism. Barter-exchange incentive is easy to be implemented, but a peer needs to spend time to find the proper peers to make mutual data exchanges, and the user has no motivation to share a file with other peers once that file's download completes.

Another kind of incentive is based on virtual currency. The system first establishes an account for each user, then it charges users for every download and rewards them for every upload. Researchers have devised many virtual currency incentive mechanisms, such as Micro-payment [3], Dandelion [4], Scrip [5], Pace [6] and so on. Compared to barter-exchange, the virtual currency incentive mechanism enables data trade between any two peers hence saves the time of searching for proper counterparty. But in the incentive mechanisms mentioned above, the system has to track every occurred trade and modify the users' account balance accordingly, which adds extra load into the system.

At the beginning, the P2P system was just used to share files, recently the P2P media streaming systems $[7,8,9]$ are obtaining a growing number of viewer population in a short period of time. The media streaming system has characteristics that different from traditional P2P file sharing system. Firstly, in a media streaming system, the peer with an advanced playback point can provide data for a behindhand peer but the latter has nothing to reciprocate, so the traditional barter exchange incentive mechanism is not suitable. Secondly, the real-time characteristic of the media streaming requires that the action of peers' searching, trading and data transmission as quick as possible, and the extra load the incentive mechanism brings into the system should be as light as possible. Thirdly, a media streaming system usually contains thousands of video files, and users scatter into these numerous video channels so it is difficult for them to cooperate [10].

Media streaming systems can be subdivided into two groups: live streaming and Video-on-Demand (VoD). Live streaming allows video content to be transmitted in real time to all requesting users. $\mathrm{VoD}$ systems provide more flexible and convenient service to users by allowing them to watch any kind of video at any point in time. VoD systems need to accommodate a large number of users watching the same video asynchronously, and watching different part of the same video at any given time. So the challenge of providing VoD services with P2P networks lies in alleviating the burden of the server, which determines if the VoD system can satisfy users' demand and keep stability as well as extensibility.

Most of the P2P VoD systems require each user to contribute a small amount of storage instead of only the playback buffer in memory as in the P2P live streaming systems. In this case, users can upload videos which have been downloaded before when they are watching another different movie, or just help other video channels which are short of uploading resources by downloading that video and 
relay it if there is room on the hard disc. This is referred as Multiple Video Cache (MVC) strategy [11, 12]. Compared with Single Video Caching (SVC), MVC could greatly improve the cooperation and alleviate the burden of the server [13].

In this paper, we propose a new incentive mechanism for $\mathrm{P} 2 \mathrm{P}$ VoD systems based on virtual currency. In our system, the server plays the role of the central bank and keeps an account for each user. Peer gains money if he shares a video file he has already completely downloaded before, and the audience members watching that video would together chip in to pay for the upload peer. We do not take into consideration the data exchange among the audience members watching the same video. Neglecting the trade among audience peers lightens the burden of the bank server and also encourages users to share the video files which they have downloaded completely.

The currency-based lightweight P2P VoD incentive mechanism we proposed has such features:

- Virtual currency produces incentive to the entire system rather than a single file. A peer can use the money he earned by contribution to enjoy any other video programs. In order to accumulate more money for their future benefits, peers would voluntarily prefer to store and share the video files they have seen, and stay online even when they are not watching any video program, which increases the cooperation ability of peers.

- The incentive mechanism only records the data transferred between upload peers and audience peers. Technically omitting the data exchanges among the audience peers largely decreases the system cost brought by the incentive mechanism.

- Our incentive mechanism encourages users to share the video files which they have downloaded completely. Compared with the audience peers who may leave the system or use VCR actions at any time, the upload peers with completed video files are more reliable and stable.

The remainder of this paper is organized as follows: in section II we introduce the currency-based lightweight incentive mechanism, in section III we analyze the incentive mechanism by game theory, and we make a conclusion in Section IV.

\section{LIGHTWEIGHT CURRENCY-BASED INCENTIVE MECHANISM}

In this section we would describe our incentive mechanism more specifically. We first briefly introduce the system structure of the $\mathrm{P} 2 \mathrm{P} \mathrm{VoD}$ system, then propose the pricing schemes of our incentive mechanism, and at last illustrate the dynamic mechanisms of the system.

\section{A. System Structure}

The P2P VoD system comprises two main modules: servers and peers. The server is responsible for providing a centralized management of the system, providing video content, and playing the role of the central bank in our incentive mechanism. On the other hand, peers in the VoD system can start watching a movie at any point of time, with small start-up times and sustainable playback rates. The structure of the P2P VoD system is shown in Fig.1.

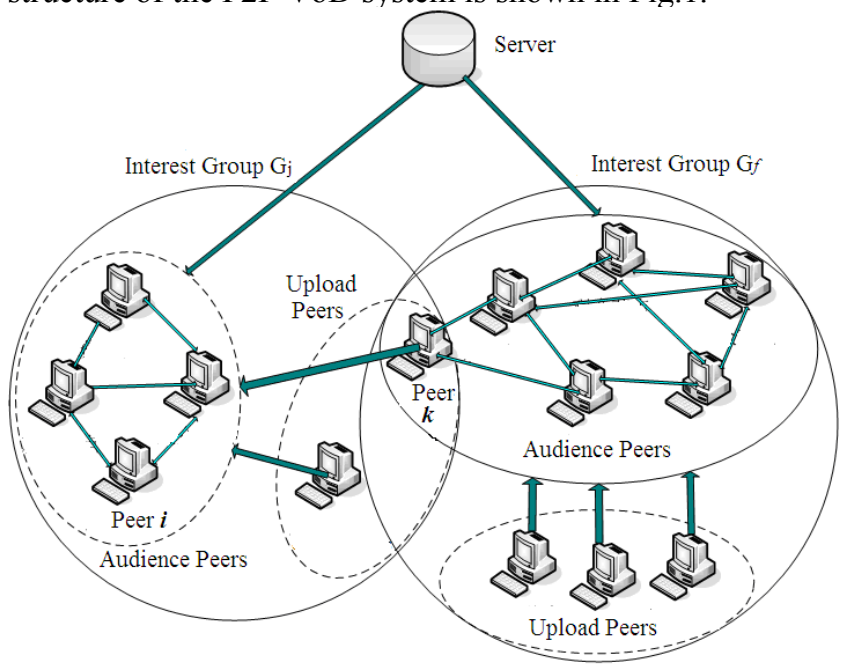

Figure 1. P2P VoD system architeccture.

As Fig. 1 shows, there are multiple video programs in the system. All the peers watching or sharing the same video file constitute a video interest group. In an interest group, peers can be further subcategorized into two categories: the audience peers which are in the course of watching the video, and the set of peers named upload peers which have already watched the video and just share the video data for the audience peers. Considering that in $\mathrm{MVC}$, one peer can watch some video program and at the same time store and share multiple video programs, so a peer can participate in multiple video interest groups. For example, in Fig.1, peer $k$ is an upload peer of interest group $G_{j}$, and also an audience peer of interest group $G_{f}$.

\section{B. Pricing Scheme}

Pricing scheme is a basic issue of constructing a monetary system: we should first decide what to price and then how to set the price. As we discussed in section I, the key problem of a P2P VoD system is to alleviate the burden of the server without degrading the playback quality of the peers. A light load of the server means a stable and economical system. So we want to construct a pricing scheme which can represent the contribution or burden of a peer bring to the server, which means that actions offloading the server would be rewarded and increasing server's burden would be charged accordingly.

We observe that in a video interest group, the upload peers play a very important part in saving server's bandwidth: an interest group with no upload peer would definitely needs the server to transmit the video data. Also, compared with an audience peer, we get that the former is superior to the latter. For an audience peer, it is possible that the peer has VCR actions, and probably cannot finish the file. So we suppose that the contribution of the upload peer is more than the audience peer when the upload bandwidth is the same. 
The data sharing between audience peers are also important, but the system would have to bear a very large cost if we record each trade and refresh the account balance of the buyer and seller accordingly. Also, in an interest group, an audience peer could download data from other audience which have earlier playback points, and at the same time offers data for the peers with lagged playback points. If we consider in a long run, an audience peer shares as much as he gets in the system. So we ignore the data exchange among audience peers and only focus on the data transfer from the upload peers to the audience peers.

We take a video interest group $G_{j}$ as an example to illustrate our pricing scheme. For simplicity, we introduce the following notations:

- $\quad P:$ the set of peers in the system;

- $\quad n:$ the number of peers in the system;

- $F$ : the set of video files in the system;

- $m$ : the number of video files in the system;

- $G_{j}$ : the interest group of video file $j, j \in F$;

- $n_{j}$ : the number of peers in the interest group $G_{j}$;

- $n_{j}^{u}$ : the number of upload peers in $G_{j}$;

- $U_{j}$ : the set of upload peers in $G_{j}$;

- $A_{j}$ : the set of audience peers in $G_{j}$;

- $\quad n_{j}^{a}$ :The number of audience peers in $G_{j}$;

- $\quad x_{i j}$ : the download rate of peer $i$ downloading video file $j, i \in A_{j}$;

- $\quad y_{k j}$ : the rate of peer $k$ uploading video file $j, k \in U_{j}$;

- $\quad z_{i j}$ : the rate of peer $i$ uploading video file $j, i \in A_{j}$;

- $\quad R_{k j}$ : the reward of peer $k$ gets from uploading file $j$, $k \in U_{j}$;

- $\quad B_{i j}:$ the bill of peer $i$ have to pay for watching video file $j, i \in A_{j}$;

- $\quad p$ : the unit price per upload bandwidth;

- $U_{i}$ : the utility of peer $i, i \in P$;

- $d_{i}$ : the disk space used in peer $i, i \in P$.

We employ "point" as our unit of currency, and we set an upload bandwidth price denoted as $p$ which is a global constant (for example, we can set $p$ as 10 points per KB). We begin with observing the upload peer $k$ of $G_{j}$. The reward peer $k$ gets from $G_{j}$ can be calculated:

$$
R_{k j}=p^{*} y_{k j}
$$

So the total reward of the set of upload peers is:

$$
R_{j}=\sum_{k=1}^{n} R_{k j}=\sum_{k=1}^{n}\left(p^{*} y_{k j}\right)=p^{*} \sum_{k=1}^{n} y_{k j}
$$

In this trade, the seller is the upload peer set and the buyer is the audience set. So we can get:

$$
B_{j}=R_{j}
$$

Since the audience peers all benefit from the contribution of upload peers, they split the bill on average. So each audience peer's payment is:

$$
B_{i j}=B_{j} / n_{j}^{a}=R_{j} / n_{j}^{a}=\left(p^{*} \sum_{k=1}^{n} y_{k j}\right) / n_{j}^{a}
$$

Notice that the price of upload bandwidth is a global uniform price, so the reward an upload peer gets only depends on the bandwidth he actually shares, and the reward has nothing to do with what video file he shares. While the payment of an audience peer depends on the number of audience in that video interest group. We can see from (4) that the payment decreases as the number of audience increases. This is easy to be understood: audience peers in a popular video usually have better cooperation between each other and needs less support by server. So the payment of watching a popular video is cheaper than an unpopular video.

Under such incentive mechanism, collusion action is not profit. Collusion peers perform upload and download in pairs to get award. But in our incentive mechanism, the pair's total income is zero if there are only these two peers in the interest group; if there are more than two audience peers in the system, the peer disguising as an audience has to watch and share the data with other audience peers, but the income is the balance between the reward and payment, which is less than the two peers just act as upload peers.

Besides, under such pricing scheme, a peer may want to use all his upload bandwidth to upload files which he has already had to maximize his income. In order to prevent such case, the system should force audience peers to upload for other audience with a certain minimum bandwidth.

\section{System Mechanisms}

In this part we introduce the system's dynamic behaviors related to the incentive mechanism.

1) A new peer joins in: When a new peer joins in the system, The bank server opens an new account and offers a freshman subsidy to help the new member downloading and watching the first video as a seed capital. The amount of the subsidy should be carefully designed: if it is set too small, the new peer could not afford to download a complete file, while if the subsidy is too large, whitewashers which leave the system and re-join the system as a new ID would take advantage of the policy and simply make no contribution to the system. A reference value to set the subsidy is the median value of all the video files' market watching price.

2) A request for a video chunk: The detailed process of a request is as follow: a peer $i$ chooses a video interest group $G_{j}$ to join in as a audience peer. The peer $i$ sends a request for a certain chunk of video to the server, and the server replies $i$ with a list of the peers in the video group and $i$ chooses from the list the audience peers which can offer data for him. If there is no suitable audience peer, peer $i$ searches the upload peer list. And if no upload peer can afford him, peer $i$ has to request for the server to transfer the data for him.

3) End of a video chunk transmission: After a video chunk is successfully transmitted from the upload peer to 
audience, the server records the interest group $G_{j}$, the upload peer $k$ and his upload bandwidth $y_{k j}$. The bank server records every trade and deposits the rewards in the account of the seller $k$ and draw points from each accounts of the buyer.

\section{GAME THEORETIC EVALUATION}

In this section, we will analyze our lightweight currencybased incentive mechanism from a game theoretic perspective. We continue to use the notations listed in Section II.

In game theory, a game consists of such components: (1) a set of players; (2) a set of strategies for each player; (3) a utility function that gives the players' utility to each list of the players' strategies.

We know that the peers in the P2P system are selfish and rational, so we deem each peer in the system as a player of the game. We also assume that every player's belief about the other players' strategies is correct. In a VoD system, the parameters a peer can decide includes: which video group to join in, the download and upload rate, and the usage of disk space to get a maximum of his utility. Other network parameters such as bandwidth, delay and jitter are also very important, we suppose in this paper that the delay and jitter are in a negative correlation with the download bandwidth so we can use the bandwidth to represent the other performance parameters for simplicity.

We define $S_{i}$ is the strategy of peer $i$, and we choose the download rate, upload rate and the amount of disk space as its variables, so $S_{i}$ could be denoted as $S_{i}=\left(x_{i}, y_{i}, d_{i}\right)$. The strategy profile of the game is the list of all players' strategies $S=\left(S_{1}, S_{2}, . ., S_{i}, . ., S_{n}\right)$.

\section{A. P2P VoD system with no incentive mechanism}

As a comparison with our schemes, we first analyze the P2P VoD system with no explicit incentive mechanism.

Now we study the utility function of the players. In the VoD system with no incentive, the utility of peer $i$ could be expressed as $U_{i}\left(x_{i}, y_{i}, d_{i}\right)$. We analyze the relation of the utility function with these variables one by one: the increasing of download rate $x_{i}$ results in small start-up delays and sustainable playback rates which brings positive utilities; while uploading files consumes the limited network resources of the user which decrease user's utility; in the VoD system, downloaded files which have been played have little value for users and occupy the disk storage. So we assume that the utility function is continuous, strictly increasing in download rate $x_{i}$ and strictly decreasing in the upload rate $y_{i}$ and disk usage amount $d_{i}$.

We next analyze the system with Nash equilibrium theory. It is obvious that the Nash equilibrium of the game is $S=\left(\left(x_{\max }, y_{\text {min }}, d_{\text {min }}\right), \ldots,\left(x_{\max }, y_{\text {min }}, d_{\text {min }}\right)\right)$; now we prove it. We consider a player $i \in P$ in the game. Assume that other players besides player $i$ follow strategy $S=\left(x_{\max }, y_{\min }, d_{\min }\right)$, in order to maximize his own utility, player $i$ would choose the strategy $S_{i}=\left(\max \left(x_{i}\right), \min \left(y_{i}\right), \min \left(d_{i}\right)\right)$. No matter what strategy other player choose, the strategy of $S_{i}=\left(\max \left(x_{i}\right), \min \left(y_{i}\right), \min \left(d_{i}\right)\right)$ is a dominant strategy for $i$, because any other strategy results in an inferior utility regardless of other peers' strategies. So the strategy $S=\left(\left(x_{\max }, y_{\min }, d_{\min }\right), \ldots,\left(x_{\max }, y_{\min }, d_{\min }\right)\right)$ is a dominant strategy and is unique.

\section{B. Lightweight currency-based incentive mechanism}

Bringing virtual currency into the system alters the function of peers' utilities. In our lightweight currency based incentive mechanism, The utility function of player $i$ can be written as $U_{i}\left(x_{i j}, y_{i}, z_{i}, d_{i}\right)$.

In order to simplify the analysis, we make an approximation version of the utility function by breaking the complex variable function into single variable functions. We get the approximate express of utility function as follow:

$$
U_{i}=f\left(x_{i j}\right)-g\left(y_{i}+z_{i j}\right)-h\left(d_{i}\right)+R_{i}-B_{i j}
$$

If we make a further substitution, we can get:

$$
U_{i}=f\left(x_{i j}\right)-g\left(y_{i}+z_{i j}\right)-h\left(d_{i}\right)+p^{*} y_{i}-\left(p^{*} \sum_{i=1}^{n} y_{i j}\right) / n_{j}^{a}
$$

We suppose that the single variable functions above are all continuous, strictly concave and strictly increasing.

We first analyze variable $z_{i j}$. As we mentioned in Section II $B$ ), the system should set a minimum upload bandwidth to force the data exchange among audience peers. We suppose that the minimum value is $z_{\min }$, then it is clear that when $z_{i j}=z_{\min }$, the utility could achieve its max. So we substitute $z_{i j}$ with the constant $z_{\min }$ in the utility function.

We now turn to the study of the disk usage variable $d_{i}$. We assume an extreme condition when $d_{i}=0$, which means no disk is used and no file is stored, thus the upload rate $y_{i}=0$ because the peer has nothing to share, and the peer's income is zero. On the other extreme condition, if $d_{i}$ equals to the total disk storage of peer $i$, the negative item of the utility function $-h\left(d_{i}\right)$ would greatly decrease user's satisfaction. So we use $d_{i}^{*}$ to denote the fixed point of $d_{i}$ which maximize the utility function.

Based on the analysis above, we can rewrite (6) as:

$$
U_{i}=f\left(x_{i j}\right)+\left(p^{*} y_{i}-g\left(y_{i}+z_{\min }\right)\right)-\left(p^{*} \sum_{i=1}^{n} y_{i j}\right) / n_{j}^{a}-h\left(d_{i}^{*}\right)
$$

In our incentive mechanism, the cost for uploading as an upload peer is less than the income it gets, and rational peers would only join in a video interest group if the utility of watching that certain video is worth its cost. So we have the inequalities below:

$$
\begin{gathered}
p^{*} y_{i}-g\left(y_{i}+z_{\min }\right)>0 \\
f\left(x_{i j}\right)-\left(p^{*} \sum_{i=1}^{n} y_{i j}\right) / n_{j}^{a}>0
\end{gathered}
$$


Now we analyze the Nash equilibrium of the system. Inequality (8) shows that the increase of upload rate can bring positive growth of a user's utility, so a peer has incentive to upload as much as possible in order to maximize his utility, thus the largest upload rate peer $i$ could achieve denoted by action $\max \left(y_{i}\right)$ dominants other actions. Inequality (9) states that the more users download, the better utility they can get, thus the maximum download rate of peer $i$ denoted as $\max \left(x_{i j}\right)$ dominants other actions no matter what strategies other player use. So the strategy profile $S=\left(\left(\max \left(x_{1}\right), \max \left(y_{1}\right), z_{\min }, d_{1}^{*}\right), . .,\left(\max \left(x_{i}\right), \max \left(y_{i}\right), z_{\min }, d_{i}^{*}\right), \ldots\right)$ is the dominant strategy of the game and it is unique.

\section{CONCLUSION AND FUTURE WORK}

In this paper, we first analyze the features of the P2P VoD system and point out that the fundamental problem of the VoD system lies in the heavy load of server and the difficulty to cooperate among peers caused by the random action and VCR functions of the peers. Then we propose a new lightweight incentive mechanism based on currency. A peer gets paid if he shares the video file he completely downloaded with other audience peers in the process of watching that video, and if the peer watches a video, he has to pay money to the upload peers together with other audience peers in that video interest group. We neglect the data exchanges among the audience peers and only record the data exchanges between upload peers and audience peers. Such pricing scheme can stimulate peers to share the video files downloaded completely which have a more stable performance. We demonstrate through game theoretical analysis that the lightweight currency-based incentive mechanism can lighten the burden of the server and increase the cooperation of peers.

For our future work, we would consider to bring differentiate service levels into the system, for example, peers with higher points could enjoy the service of higher resolution of videos. Differentiated service could further stimulate the peers in the system to share more in order to get better service.

Currency control of the system is also an open problem. If users own too much currency, they could easily get high quality services hence have less incentive to share, while if the currency is in a shortage, some peers would be too poor to afford any video and become bankruptcy. In our future work, we would like to use macroeconomic theory to analyze the influence of currency to the system dynamics and manage the currency in the system to control it in a suitable level.

Moreover, we would also take ISP-friendly into considerations as our next work. We would study how cross-
ISP traffic can be reduced while maintaining the performance of the VoD system. Another idea is for the ISPs to join in the system and get paid according to the link utilization of the network.

\section{ACKNOWLEDGMENT}

This work was supported by the National Natural Science Foundation of China (No.60502037), the Specialized Research Fund for the Doctoral Program of Higher Education (No. 200800131019), the Program for New Century Excellent Talents in University (No.NECT-070109), and the National High-Tech Research and Development Plan of China (No.2007AA01Z206).

\section{REFERENCES}

[1] D. Hughes, G. Coulson, and J. Walkerdine, "Free riding on Gnutella revisited: The bell tolls?" IEEE Distributed Systems Online, vol. 6, no. 6,2005 .

[2] B. Cohen, "Incentives build robustness in BitTorrent," Workshop on Economics of Peer-to-Peer Systems, 2003.

[3] P. Golle, K. Leyton-Brown, and I. Mironov, "Incentives for sharing in peer-to-peer networks," ACM Conference on Electronic Commerce, 2001, pp. 264-267.

[4] M. Sirivianos, J. Park, X. Yang, and S. Jarecki, "Dandelion: cooperative content distribution with robust incentives." USENIX Annual Technical Conference on Proceedings of the USENIX Annual Technical Conference, 2007.

[5] I. A. Kash, E. J. Friedman, and J. Y. Halpern, "Optimizing scrip systems: efficiency, crashes, hoarders, and altruists." Proceedings of the 8th ACM Conference on Electronic Commerce. EC '07. ACM, New York, NY, 305-315.

[6] C. Aperjis, M. J. Freedman, and R. Johari. "Peer-Assisted Content Distribution with Prices." Proc. ACM SIGCOMM Conference on emerging Networking EXperiments and Technologies(CoNext '08) Madrid, Spain, December 2008.

[7] "GridCast", http://www.gridcast.cn/.

[8] "PPStream", http://www.ppstream.com/.

[9] "PPlive", http://www.pplive.com/.

[10] B. Cheng, L. Stein, H, Jin, X, Liao, and Z. Zhang, "GridCast: Improving peer sharing for P2P VoD." ACM Transactions on Multimedia Computing Communications and Applications. Oct. 2008. 4(4): 1-31.

[11] B. Cheng, L. Stein, H, Jin, X, Liao, and Z. Zhang, "GridCast: Improving peer sharing for P2P VoD." ACM Transactions on Multimedia Computing Communications and Applications. Oct. 2008. 4(4): 1-31.

[12] Y. Huang, T. Z. Fu, D. Chiu, J. C. Lui, and C. Huang, "Challenges, design and analysis of a large-scale p2p-vod system". Proceedings of the ACM SIGCOMM 2008 Conference on Data Communication Seattle, USA, August, 2008.

[13] D. Wu, C. Liang, Y. Liu and K. Ross,"View-Upload Decoupling: A Redesign of Multi-Channel P2P Video Systems", Proceedings of IEEE Conference on Computer and Communications (INFOCOM) Mini-Conference, 2009. 